\title{
A Novel Methicillin-Resistant Staphylococcus aureus t11469 and a Poultry Endemic Strain t002 (ST5) Are Present in Chicken in Ebonyi State, Nigeria
}

\author{
Amos Nworie, ${ }^{1,2,3,4}$ Azi S. Onyema, ${ }^{1}$ Simon I. Okekpa, ${ }^{1}$ Michael O. Elom, ${ }^{1}$ Nse O. Umoh, \\ Victor U. Usanga, ${ }^{1}$ Gideon A. Ibiam, ${ }^{1}$ Boniface N. Ukwah, ${ }^{1}$ Lynda C. Nwadi, \\ Chinwe Ezeruigbo, ${ }^{5}$ Busayo O. Olayinka, ${ }^{2}$ Joseph O. Ehinmidu, ${ }^{2}$ Josiah A. Onaolapo, ${ }^{2}$ \\ Blake M. Hanson, ${ }^{3,4}$ Shylo E. Wardyn, ${ }^{3,4}$ and Tara C. Smith ${ }^{6}$ \\ ${ }^{1}$ Department of Medical Laboratory Science, Faculty of Health Sciences, Ebonyi State University, Abakaliki, Nigeria \\ ${ }^{2}$ Department of Pharmaceutics and Pharmaceutical Microbiology, Faculty of Pharmaceutical Sciences, \\ Ahmadu Bello University, Zaria, Nigeria \\ ${ }^{3}$ Department of Epidemiology, University of Iowa, Iowa City, IA, USA \\ ${ }^{4}$ Center for Emerging Infectious Diseases, University of Iowa, College of Public Health, Iowa City, IA, USA \\ ${ }^{5}$ Department of Nursing Science, Faculty of Health Science, Ebonyi State University, Abakaliki, Nigeria \\ ${ }^{6}$ Department of Biostatistics, Environmental Health Sciences, and Epidemiology, Kent State University, Kent, OH, USA
}

Correspondence should be addressed to Tara C. Smith; tsmit176@kent.edu

Received 6 September 2017; Revised 23 October 2017; Accepted 29 October 2017; Published 19 November 2017

Academic Editor: Paola Di Carlo

Copyright (c) 2017 Amos Nworie et al. This is an open access article distributed under the Creative Commons Attribution License, which permits unrestricted use, distribution, and reproduction in any medium, provided the original work is properly cited.

\begin{abstract}
Background. The changing epidemiology of methicillin-resistant Staphylococcus aureus (MRSA) from a hospital-associated pathogen to an organism commonly found in the community and in livestock reflects an organism well-equipped to survive in diverse environments and adjust to different environmental conditions including antimicrobial use. Methods. We investigated the molecular epidemiology of S. aureus and MRSA in poultry in Ebonyi State, Nigeria. Samples were collected from 1800 birds on 9 different farms within the state. Positive isolates were tested for antibiotic susceptibility and molecular typing. Results. Prevalence in birds was $13.7 \%(247 / 1800)$. MRSA prevalence in poultry was $0.8 \%$. The prevalence of MRSA in broilers and layers was $1.2 \%$ and $0.4 \%$, respectively. All tested isolates were susceptible to vancomycin. Molecular analysis of the isolates revealed 3 spa types: t002, t084, and a novel spa type, t11469. The novel spa type t11469 belonged to sequence type ST5. Conclusion. The detection of t002 in chicken suggests the presence of livestock-associated MRSA in poultry in Ebonyi State. The detection of the new spa type t11469 in poultry that has not been characterised to ascertain its pathogenic potential remains a cause for concern, especially as some were found to carry PVL genes, a putative virulence factor in staphylococcal infection.
\end{abstract}

\section{Introduction}

Staphylococcus aureus is a known commensal of both man and animals and methicillin-resistant Staphylococcus aureus (MRSA) remains a major hospital and community pathogen [1-3]. Diseases associated with $S$. aureus include osteomyelitis, pneumonia, meningitis, arthritis, endocarditis, septicemia, deep tissue abscesses, skin, and soft-tissue infections (SSTIs), as well as toxic shock syndrome, among others $[1,4,5] . S$. aureus is also a common cause of wound and urinary tract infections $[6,7]$.

S. aureus has been detected in several species of animals and products from animals [8-12], including poultry. The cloacae and nostrils of poultry remain important sites for the recovery of S. aureus and MRSA [13]. Intensity of contact with live animals is documented a risk factor for MRSA colonization [14]. While colonization alone does not harm the host, livestock-associated MRSA has also been reported to cause 
severe infections in humans, including endocarditis $[15,16]$. However, while studies have been carried out investigating livestock-associated S. aureus in Europe, North America, and Asia, relatively few studies have been carried out in Africa. This study investigated the presence of $S$. aureus, including potential livestock-associated types, in poultry in Ebonyi State, Nigeria.

\section{Materials and Methods}

2.1. Sample Collection and Culture. Cloacae and nasal samples from 1800 birds (900 broilers and 900 layers) were collected using sterile swabs. Birds were selected at random from 9 poultry farms in the 3 senatorial districts in Ebonyi State, Nigeria (3 farms from each senatorial district). Swabs were processed within 24 hours by inoculation into $6.5 \%$ $\mathrm{NaCl}$ Staph enrichment broth. Isolation and identification of $S$. aureus were achieved through the use of mannitol salt plates, CNA plates with 5\% sheep blood, and ChromAgar plates (CHROMagar, France). Isolates were confirmed as $S$. aureus using catalase and coagulase tests and further confirmed by Staph Latex Agglutination test (Pastorex Staphplus, Bio Rad). Animal owners consented to testing of farmowned poultry.

2.2. Molecular Testing. S. aureus isolates were tested for the Panton-Valentine leukocidin (PVL) genes, methicillin resistance gene (mecA), and staphylococcal protein A (spa) gene as previously described [17]. Multilocus sequence typing (MLST) was carried out as described by Enright et al. [18] and the detection of the tetracycline resistance gene tetM as described by Weigel et al. [19], and SCCmec typing was performed according to [20].

2.3. Antibiotic Susceptibility Testing. The resistance of isolates to a panel of 11 antibiotics was determined by the KirbyBauer-Clinical and Laboratory Standards Institute (CLSI) modified disc agar diffusion (DAD) method [21]. Isolates were tested for resistance to the following antibiotics: augmentin, cefoxitin, cefuroxime, chloramphenicol, doxycycline, erythromycin, gentamycin, levofloxacin, tetracycline, trimethoprim/sulfamethoxazole, and vancomycin.

2.4. Statistical Analysis. The data obtained were analyzed by ANOVA using the statistical package for social sciences (SPSS) version 20.2, Chicago, Illinois, USA. A p value of 0.05 or less was considered statistically significant.

\section{Results}

3.1. Prevalence of S. aureus. A total of 1800 birds consisting of 900 broilers and 900 layers from 9 farms in Ebonyi State were swabbed. S. aureus was recovered from the cloacae and nostrils of both broilers and layers. The number of broilers and layers positive for S. aureus was 122 (13.5\%) and 125 $(13.8 \%)$, respectively. The overall prevalence of $S$. aureus was 247/1800 (13.7\%); see Table 1 . Of the birds where S. aureus was recovered, $52.2 \%(129 / 247)$ of the colonized birds were positive in both the cloacae and nostrils, while $27.1 \%$ (67/247)
TABLE 1: Percentage prevalence of $S$. aureus in broilers and layers per farm.

\begin{tabular}{lccc}
\hline Farm \# & $\begin{array}{c}\text { Layers, \# (\%) } \\
\text { positive }\end{array}$ & $\begin{array}{c}\text { Broilers, \# (\%) } \\
\text { positive }\end{array}$ & $\begin{array}{c}\text { Overall, \# (\%) } \\
\text { positive }\end{array}$ \\
\hline Farm 1 & $19(19.0 \%)$ & $14(14.0 \%)$ & $33(16.5 \%)$ \\
Farm 2 & $13(13.0 \%)$ & $18(18.0 \%)$ & $31(15.5 \%)$ \\
Farm 3 & $11(11.0 \%)$ & $19(19.0 \%)$ & $30(15.0 \%)$ \\
Farm 4 & $11(11.0 \%)$ & $12(12.0 \%)$ & $23(11.5 \%)$ \\
Farm 5 & $17(17.0 \%)$ & $8(8.0 \%)$ & $25(12.5 \%)$ \\
Farm 6 & $10(10.0 \%)$ & $13(13.0 \%)$ & $23(11.5 \%)$ \\
Farm 7 & $19(19.0 \%)$ & $16(16.0 \%)$ & $35(17.5 \%)$ \\
Farm 8 & $12(12.0 \%)$ & $10(10.0 \%)$ & $22(11.0 \%)$ \\
Farm 9 & $10(10.0 \%)$ & $15(15.0 \%)$ & $25(12.5 \%)$ \\
\hline Total & $122(12.2 \%)$ & $125(12.5 \%)$ & $247(13.7 \%)$ \\
\hline
\end{tabular}

100 broilers and 100 layers were sampled on each farm.

TABLE 2: Antibiotic resistance of poultry isolates.

\begin{tabular}{lc}
\hline Antibiotic & $\#(\%)$ resistant \\
\hline Augmentin & $2(0.8 \%)$ \\
Cefoxitin & $15(6.1 \%)$ \\
Cefuroxime & $13(5.3 \%)$ \\
Chloramphenicol & $30(12.1 \%)$ \\
Doxycycline & $19(7.7 \%)$ \\
Erythromycin & $48(19.4 \%)$ \\
Gentamycin & $13(5.3 \%)$ \\
Levofloxacin & $2(0.8 \%)$ \\
Tetracycline & $113(45.7 \%)$ \\
Trim/sulf & $101(40.9 \%)$ \\
Vancomycin & $0(0 \%)$ \\
\hline
\end{tabular}

were positive only in the cloacae, and $20.6 \%$ (51/247) of the colonized birds were positive only in the nostrils.

The percentage prevalence of broilers and layers varied between farms but were similar overall. Total prevalence ranged from $11.0 \%$ to $16.5 \%$, in broilers, from $10.0 \%$ to $19.0 \%$, and in layers, from $8.0 \%$ to $19.0 \%$ (Table 1 ).

Statistical analysis using ANOVA of birds with $S$. aureus in both the cloacae and nostrils, cloacae alone, and nostrils alone showed that there was a significant difference between the groups $(p \leq 0.05)$. Examining the prevalence of $S$. aureus recovered from broilers and layers showed a $p$ value of 0.85 , which was not significant.

3.2. Antibiotic Resistance. Antibiotic susceptibility testing (AST) of all the isolates $(n=247)$ was carried out against a panel of 11 antibiotics. Results showed that $S$. aureus exhibited varying degrees of susceptibility (Table 2). Over $40 \%$ of all isolates were resistant to tetracycline $(45.7 \%$ of isolates tested, 113/247 isolates) and trimethoprim/sulfamethoxazole (40.9\%; 101/247 isolates), almost $20 \%$ to erythromycin $(19.4 \%$, $48 / 247)$, and more than $10 \%$ to chloramphenicol $(12.1 \%$, $30 / 247)$. Fewer samples were resistant to doxycycline, cefoxitin, cefuroxime, gentamycin, and levofloxacin. No resistance was seen to vancomycin. 
TABLE 3: Molecular analyses of selected isolates.

\begin{tabular}{|c|c|c|c|c|c|}
\hline spa type & MSSA \# (\%) & MRSA \# (\%) & PVL & tetM & Sequence type (ST) \\
\hline t002 & $12(70.6 \%)$ & $5(29.4 \%)$ & 4 (MSSA) & & ST 5, ST 15, ST 121 \\
\hline $\mathrm{t} 084$ & $7(77.8 \%)$ & $2(22.2 \%)$ & 1 (MSSA) & 1 (MRSA) & ST 15 \\
\hline t11469 & $3(75.0 \%)$ & $1(25.0 \%)$ & 1 (MSSA) & & ST5 \\
\hline
\end{tabular}

MRSA was recovered from multiple farms. The overall prevalence of MRSA was $15 / 1800(0.8 \%)$ birds, or $6.1 \%$ of all positive isolates $(15 / 247)$. MRSA was more common in broilers $(11 / 900,1.2 \%)$ compared to layers $(4 / 900,0.4 \%)$, a statistically significant difference. MRSA ranged from $0.0 \%$ (farms 4,8 , and 9) to $21.1 \%$ of positive broiler isolates (4/19) and $5 / 33$ isolates (15.2\%) on farm 1 .

3.3. Molecular Analyses. spa typing results of 30 isolates selected equally from the 9 farms identified 3 spa types: t002, t084, and t11469. There was an overlap of the spa types between MSSA and MRSA (Table 3). Of the isolates typed, $56.7 \%(17 / 30)$ were t002 and $30.0 \%(9 / 30)$ were t084. Novel spa type t11469 accounted for the remaining $13.3 \%$ (4/30) of isolates. All spa types included more MSSA than MRSA. In spa type t002, 70.6\% of isolates of this spa type were MSSA $(12 / 17)$, while $29.4 \%(5 / 17)$ were MRSA. In spa type t084, the prevalence of MSSA was $77.8 \%$ (7/9) and MRSA $22.2 \%$ (2/9). For spa type t11469, 75.0\% (3/4) isolates were MSSA and $25.0 \%(1 / 4)$ were MRSA.

The 30 spa-typed isolates were screened for PVL and tetM; 20.0\% (6/30) were PVL-positive; 3.3\% (1/30) were positive for tetM (spa type t084, MSSA). SCCmec analyses were carried out on 6 MRSA samples ( 3 from t002, 2 from t084, and one t11469). All the MRSA tested were mecA positive and were SCCmec type V. MLST analyses showed t084 spa types belonged to ST15 and t11469 to ST5, and t002 spa types were a mix of ST5, ST15, and ST121.

\section{Discussion}

S. aureus and methicillin-resistant $S$. aureus were recovered from both broilers and layers in this study. The percentage of animals with $S$. aureus recovered from both cloacae and nostrils were higher than from cloacae and nostrils alone. This suggests that cloacae and nostrils are important sites for the recovery of $S$. aureus, a combination which gives better yield. This was consistent with earlier work in Belgium [13], which reported higher recovery of $S$. aureus from multiple sites than the sites screened singly.

All the isolates exhibited varying degrees of resistance to the panel of 11 antibiotics tested. Almost half (45.7\%) of tested samples were resistant to tetracycline. Tetracycline is the most commonly used food supplement and growth promotion factor in poultry farms in this area. Others include trimethoprim/sulfamethoxazole, chloramphenicol, erythromycin, and quinolones. These drugs are also used for disease treatment in these farms. Regulation of antibiotic use in poultry in Ebonyi state and Nigeria more broadly is lacking, which could contribute to the high levels of resistance observed in this environment.
Of 30 isolates examined, only 1 (3.3\%) was positive for the tet $M$ gene, one of the genes encoding for tetracycline resistance. The low percentage of tet $M$ recorded might suggest the involvement of other tetracycline resistance genes in tetracycline resistance observed in this study. Fluit et al. [22] have reported that tetracycline resistance is determined by several tetracycline (tet) genes, and the involvement of other tetracycline resistance genes in poultry has been reported elsewhere [23].

The percentage recovery of MRSA from broilers was $1.2 \%$ and $.4 \%$ in layers. Reasons for the differences in the recovery of MRSA from broilers and layers were not clear, as their rearing conditions were similar. Though there was no significant difference between number of $S$. aureus recovered from broilers and layers, there was a significant difference in MRSA recovered from both, though this was a comparison of small numbers and caution should be taken not to overinterpret results.

spa typing showed that 3 spa types of $S$. aureus, including a livestock-indicator spa type t002, were circulating among chicken in Ebonyi State. Lowder et al. [12] and Köck et al. [16] previously reported this spa type in poultry. A novel spa type t11469 was also detected, which to the best of our knowledge has never been characterised in Ebonyi or elsewhere. Our data showed that 6 isolates were positive for PVL gene, out of which 5 were MSSA while one was MRSA. The finding of PVL-carrying isolates in chicken may portend a serious health hazard to the poultry farm workers and their families due to possibility of farm-to-family transmission and the association of PVL with heightened virulence, though this remains controversial [24]. Furthermore, the isolation of lukS-lukF carrying isolates of $S$. aureus in this study might constitute a health risk to the general public as chicken are reared in homes and live chicken are sold on the streets and in rural markets where little attention is paid to hygiene. Prior reports have shown that PVL-positive MSSA is common in West Africa [25].

Multilocus sequence analysis showed that $S$. aureus in chicken in Ebonyi State displayed three sequence types: ST5, ST15, and ST121. ST5 had 2 spa types: t002 and t11469. Surprisingly, t002 was found in all three identified sequence types. The spa type t002 of ST5 is a livestock endemic strain thought to have left its niche as a nosocomial strain to livestock from where it is involved in human diseases [12], similar to that of another livestock-associated strain, ST398 [26]. The documentation of the novel strain, t11469, in ST5 could suggest that it could be related to t002; however, their spa repeats are quite different (t002, 26-23-17-34-1720-17-12-17-16; t11469, 04-44-24-33-31-12-16-34-12-25-22-34), suggesting divergent spa genes. 
The discovery of spa type t002 in ST15 and ST121 was intriguing, as available reports have not demonstrated t002 in these sequence types. Its presence in these sequence types could either suggest adaptation to different ecological condition, as previously reported [27], or local dispersion of spa types in different regions and environment [28], for example, by horizontal transmission of the spa gene into genetic backgrounds of ST15 and ST121 strains. Furthermore, the recovery t084 in poultry suggests an organism highly equipped to cross interspecies borders. It could also be suggestive of the movement of MRSA from its niche in human clinics to livestock as has been variously reported [29]. All MRSA isolates tested carried SCCmec type V, which has been found both in the community [30] and in livestock, including ST398 in pigs $[8,15]$.

There were important limitations to this study. Though birds within the farms were randomly selected, a convenience sample of farms participated, potentially biasing the study. However, it is unlikely that farmers had any knowledge of the $S$. aureus status of their farms prior to enrolment. Samples from individuals or the environment were not collected, and as budgetary issues allowed only a subset of positive samples to be molecularly typed (30 of 247 positive isolates), we may have missed some of the diversity in our broader sample. Further research studies should examine birds within live markets as well as on farms in order to assess risk to consumers in addition to farm workers.

\section{Conclusion}

The detection of a new spa type t11469 carrying the PVL gene could portend a health hazard if it is commonly transmitted to humans. The isolation of this new spa type in poultry especially as it has not been characterized to establish its pathogenic potential or its inherent virulence factors remains a public health threat. Additional sequencing and characterization of t11469 are necessary to establish the pathogenic potential of this novel spa type. Poultry farm workers in this environment should ensure the highest level of hygiene, including constant hand washing to prevent interspecies transfer of this new strain, or other strains of $S$. aureus that may be present on Nigerian poultry farms.

\section{Conflicts of Interest}

The authors declare no conflicts of interest.

\section{Acknowledgments}

This research was funded by Tertiary Education Trust Fund (TETfund) through Ebonyi State University, Abakaliki, Nigeria (Amos Nworie).

\section{References}

[1] N. Zetola, J. S. Francis, E. L. Nuermberger, and W. R. Bishai, "Community-acquired meticillin-resistant Staphylococcus aureus: an emerging threat," The Lancet Infectious Diseases, vol. 5, no. 5, pp. 275-286, 2005.
[2] M. M. Mwangi, W. W. Shang, Y. Zhou et al., “Tracking the in vivo evolution of multidrug resistance in Staphylococcus aureus by whole-genome sequencing," Proceedings of the National Acadamy of Sciences of the United States of America, vol. 104, no. 22, pp. 9451-9456, 2007.

[3] F. Djoudi, C. Bonura, S. Benallaoua et al., "Panton-Valentine leukocidin positive sequence type 80 methicillin-resistant Staphylococcus aureus carrying a staphylococcal cassette chromosome mec type IVc is dominant in neonates and children in an Algiers hospital," New Microbiologica, vol. 36, no. 1, pp. 4956, 2013.

[4] S. O. Jensen and B. R. Lyon, "Genetics of antimicrobial resistance in Staphylococcus aureus," Future Microbiology, vol. 4, no. 5, pp. 565-582, 2009.

[5] C. Mammina, C. Calà, C. Bonura et al., "Polyclonal non multiresistant methicillin resistant Staphylococcus aureus isolates from clinical cases of infection occurring in Palermo, Italy, during a one-year surveillance period," Annals of Clinical Microbiology and Antimicrobials, vol. 11, article no. 17, 2012.

[6] H. Sina, F. Baba-Moussa, T. A. Ahoyo et al., "Antibiotic susceptibility and toxins production of staphylococcus aureus isolated from clinical samples from benin," African Journal of Microbiology Research, vol. 5, no. 18, pp. 2797-2803, 2011.

[7] A. O. Shittu, K. Okon, S. Adesida et al., "Antibiotic resistance and molecular epidemiology of Staphylococcus aureus in Nigeria," BMC Microbiology, vol. 11, article 92, 2011.

[8] T. C. Smith, M. J. Male, A. L. Harper et al., "Methicillinresistant Staphylococcus aureus (MRSA) strain ST398 is present in midwestern U.S. swine and swine workers," PLoS ONE, vol. 4, no. 1, Article ID e4258, 2009.

[9] M. Wulf and A. Voss, "MRSA in livestock animals—an epidemic waiting to happen?" Clinical Microbiology and Infection, vol. 14, no. 6, pp. 519-521, 2008.

[10] M. Nemati, K. Hermans, U. Lipinska et al., "Antimicrobial resistance of old and recent Staphylococcus aureus isolates from poultry: first detection of livestock-associated methicillinresistant strain ST398," Antimicrobial Agents and Chemotherapy, vol. 52, no. 10, pp. 3817-3819, 2008.

[11] H. Hasman, A. Moodley, L. Guardabassi, M. Stegger, R. L. Skov, and F. M. Aarestrup, "spatype distribution in Staphylococcus aureusoriginating from pigs, cattle and poultry," Veterinary Microbiology, vol. 141, no. 3-4, pp. 326-331, 2010.

[12] B. V. Lowder, C. M. Guinane, N. L. B. Zakour et al., "Recent human-to-poultry host jump, adaptation, and pandemic spread of Staphylococcus aureus," Proceedings of the National Acadamy of Sciences of the United States of America, vol. 106, no. 46, pp. 19545-19550, 2009.

[13] D. Persoons, S. Van Hoorebeke, K. Hermans et al., "Methicillinresistant Staphylococcus aureus in poultry," Emerging Infectious Diseases, vol. 15, no. 3, pp. 452-453, 2009.

[14] B. A. G. L. Van Cleef, E. M. Broens, A. Voss et al., "High prevalence of nasal MRSA carriage in slaughterhouse workers in contact with live pigs in the Netherlands," Epidemiology and Infection, vol. 138, no. 5, pp. 756-763, 2010.

[15] M. J. Schijffelen, C. E. Boel, J. A. G. van Strijp, and A. C. Fluit, "Whole genome analysis of a livestock-associated methicillinresistant Staphylococcus aureus ST398 isolate from a case of human endocarditis," BMC Genomics, vol. 11, no. 1, article no. $376,2010$.

[16] R. Köck, F. Schaumburg, A. Mellmann et al., "Livestockassociated methicillin-resistant Staphylococcus aureus (MRSA) 
as causes of human infection and colonization in Germany," PLoS ONE, vol. 8, no. 2, Article ID e55040, 2013.

[17] T. C. Smith, W. A. Gebreyes, M. J. Abley et al., "Methicillinresistant staphylococcus aureus in pigs and farm workers on conventional and antibiotic-free swine farms in the USA," PLoS ONE, vol. 8, no. 5, Article ID e63704, 2013.

[18] M. C. Enright, N. P. J. Day, C. E. Davies, S. J. Peacock, and B. G. Spratt, "Multilocus sequence typing for characterization of methicillin-resistant and methicillin-susceptible clones of Staphylococcus aureus," Journal of Clinical Microbiology, vol. 38, no. 3, pp. 1008-1015, 2000.

[19] L. M. Weigel, R. M. Donlan, D. H. Shin et al., "High-level vancomycin-resistant Staphylococcus aureus isolates associated with a polymicrobial biofilm," Antimicrobial Agents and Chemotherapy, vol. 51, no. 1, pp. 231-238, 2007.

[20] E. Ghaznavi-Rad, M. Nor Shamsudin, Z. Sekawi, A. Van Belkum, and V. Neela, "A simplified multiplex PCR assay for fast and easy discrimination of globally distributed staphylococcal cassette chromosome mec types in meticillin-resistant Staphylococcus aureus," Journal of Medical Microbiology, vol. 59, no. 10, pp. 1135-1139, 2010.

[21] CLSI, Performance Standards for Antimicrobial Susceptibility Testing; Twenty-Third Informational Supplement, 2012.

[22] A. C. Fluit, M. R. Visser, and F.-J. Schmitz, "Molecular detection of antimicrobial resistance," Clinical Microbiology Reviews, vol. 14, no. 4, pp. 836-871, 2001.

[23] G. Huys, K. D’Haene, J.-M. Collard, and J. Swings, "Prevalence and molecular characterization of tetracycline resistance in enterococcus isolates from food," Applied and Environmental Microbiology, vol. 70, no. 3, pp. 1555-1562, 2004.

[24] M. Otto, "Community-associated MRSA: a dangerous epidemic," Future Microbiology, vol. 2, no. 5, pp. 457-459, 2007.

[25] S. Breurec, S. B. Zriouil, C. Fall et al., "Epidemiology of methicillin-resistant Staphylococcus aureus lineages in five major African towns: emergence and spread of atypical clones," Clinical Microbiology and Infection, vol. 17, no. 2, pp. 160-165, 2011.

[26] L. B. Price, M. Stegger, H. Hasman et al., "Staphylococcus aureus CC398: host adaptation and emergence of methicillin resistance in livestock," mBio, vol. 3, no. 1, p. e00305-11, 2012.

[27] A. van Belkum, N. Riewerts Eriksen, M. Sijmons et al., "Are variable repeats in the spa gene suitable targets for epidemiological studies of methicillin-resistant Staphylococcus aureus strains?" European Journal of Clinical Microbiology \& Infectious Diseases, vol. 15, no. 9, pp. 768-770, 1996.

[28] W. Ruppitsch, A. Indra, A. Stöger et al., "Classifying spa types in complexes improves interpretation of typing results for methicillin-resistant Staphylococcus aureus," Journal of Clinical Microbiology, vol. 44, no. 7, pp. 2442-2448, 2006.

[29] P. A. Hunter, S. Dawson, G. L. French et al., "Antimicrobialresistant pathogens in animals and man: prescribing, practices and policies," Journal of Antimicrobial Chemotherapy, vol. 65, supplement 1, pp. i3-i17, 2010.

[30] International Working Group on the Classification of Staphylococcal Cassette Chromosome Elements (IWG-SCC), "Classification of staphylococcal cassette chromosome mec (SCCmec): guidelines for reporting novel SCCmec elements," Antimicrobial Agents and Chemotherapy, vol. 53, no. 12, pp. 4961-4967, 2009. 


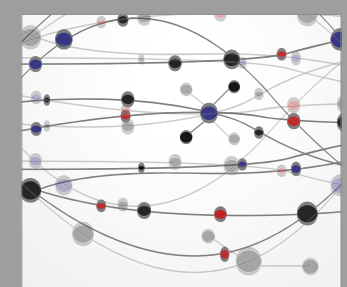

The Scientific World Journal
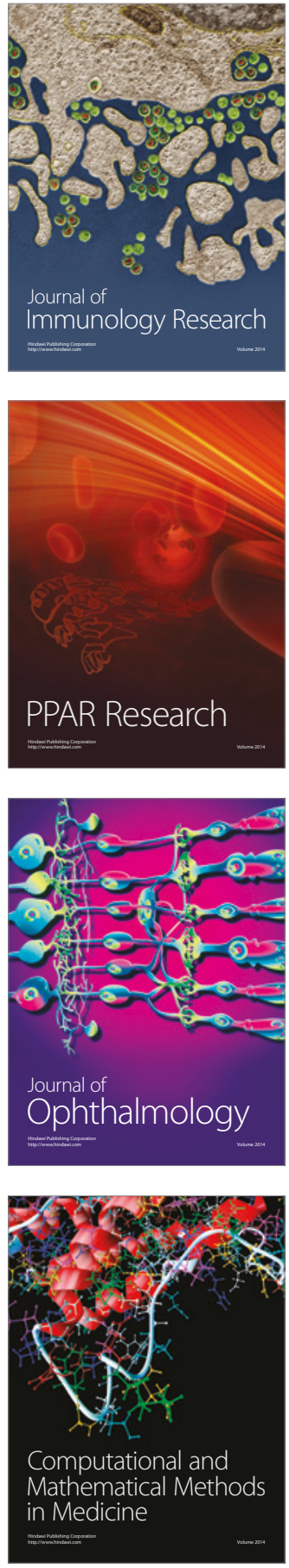

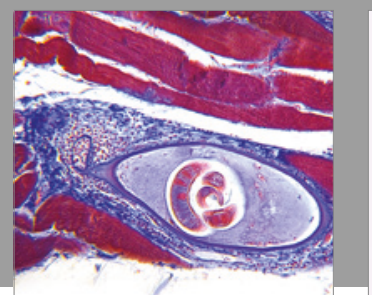

Gastroenterology Research and Practice
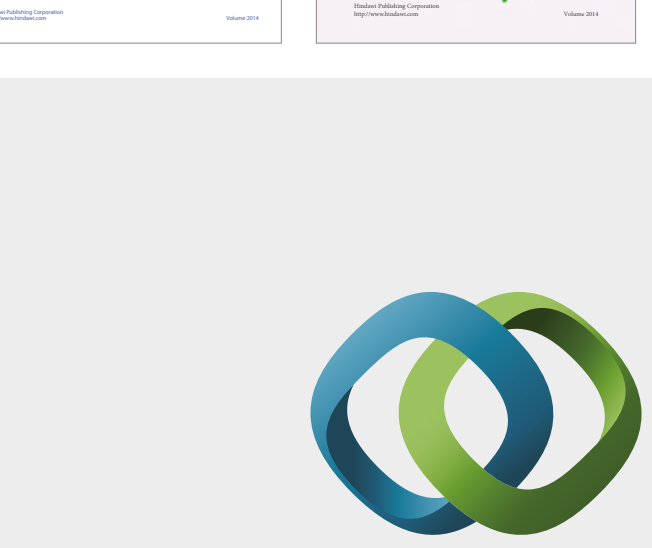

\section{Hindawi}

Submit your manuscripts at

https://www.hindawi.com
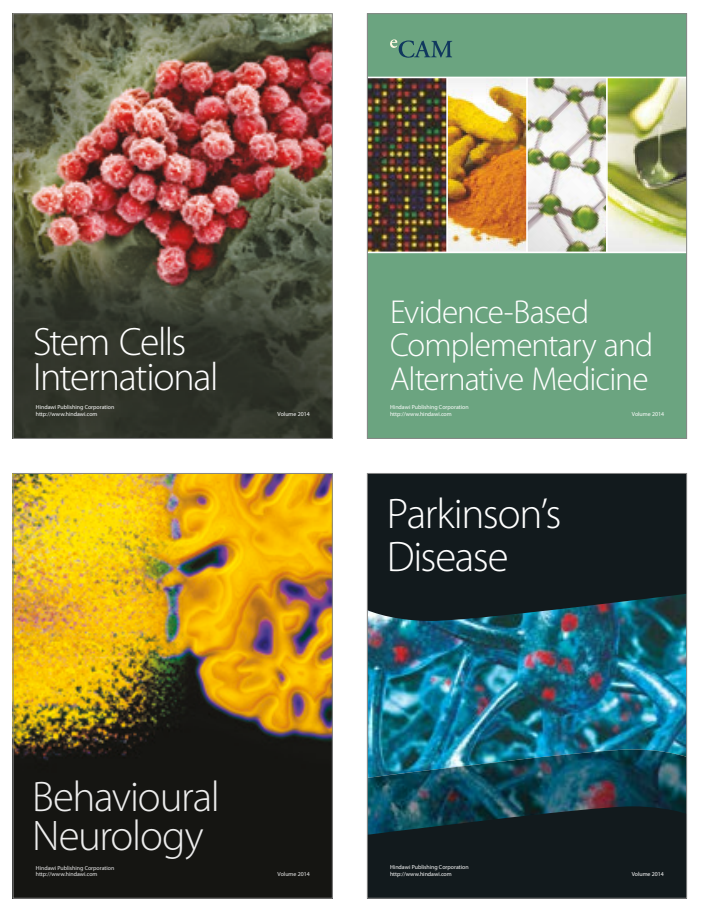
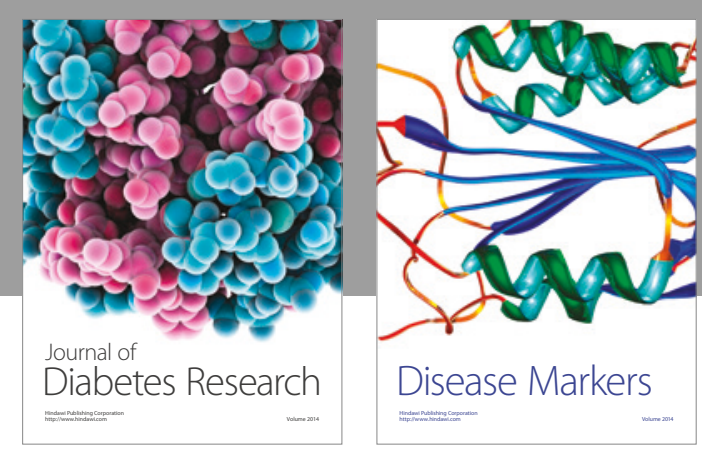

Disease Markers
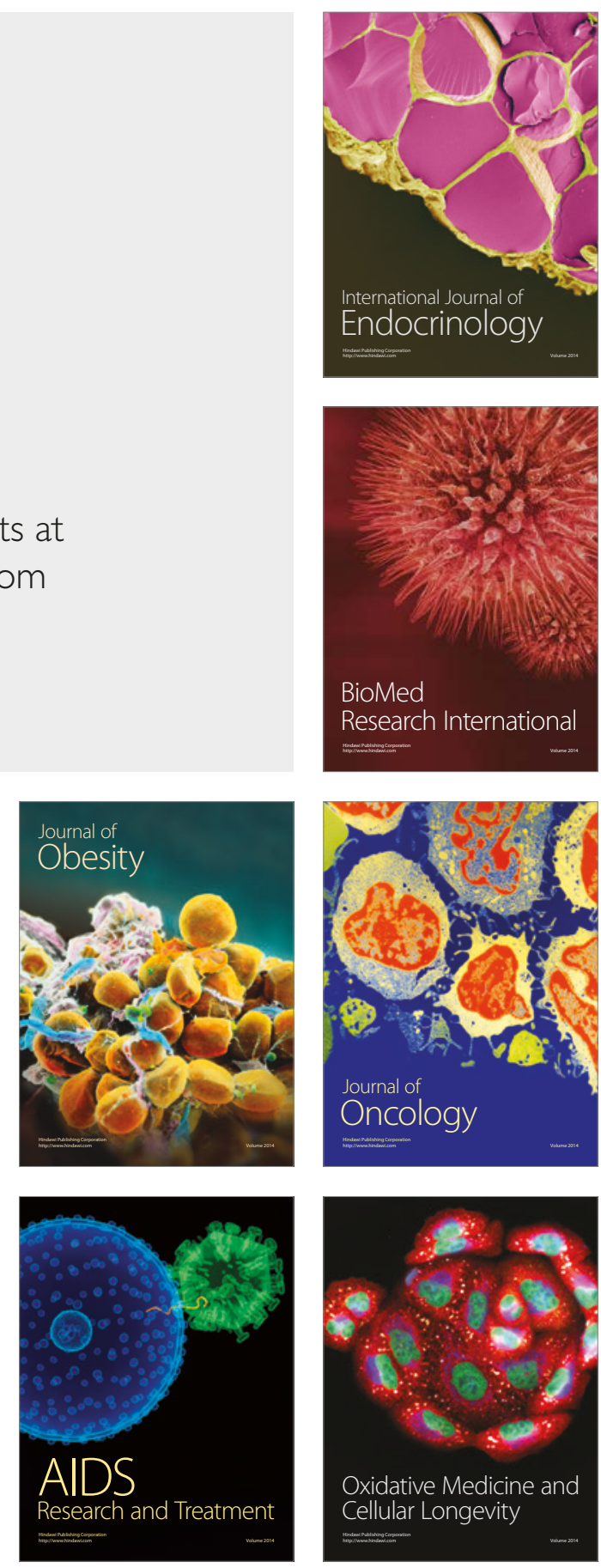